\title{
HPV-ASSOCIATED PENILE PIGMENTED LESION
}

\author{
J. Ananiev ${ }^{1}$, G. Tchernev² ${ }^{2}$ S. Philipov ${ }^{3}$, C. Guarneri ${ }^{4}$, J. W. Patterson ${ }^{5}$, L. Zisova ${ }^{6}$, R. Hristova ${ }^{6}$, \\ M. Betekhtin ${ }^{7}$ and U. Wollina ${ }^{8}$ \\ 'Department of General and Clinical Pathology, Medical Faculty, Trakia University, Stara Zagora \\ 2Polyclinic for Dermatology and Venerology, University Hospital Lozenetz, \\ "Saint Kliment Ohridski University", Sofia \\ 32Department of General and Clinical Pathology, Medical Faculty, "Saint Kliment Ohridski University", \\ ${ }^{4}$ Assistant Professor of Dermatology and Venereology, Department of Social Territorial Medicine, \\ Section of Dermatology, University of Messina, Italy \\ ${ }^{5}$ Division of Surgical Pathology and Cytopathology, University of Virginia, USA \\ ${ }^{6}$ Department of Dermatology and Venerology, Plovdiv Medical University, Bulgaria \\ ${ }^{7}$ Medical Center, Moscow, Russia \\ ${ }^{8}$ Department of Dermatology \& Allergology, Hospital Dresden-Friedrichstadt, Academic Teaching \\ Hospital of the Technical University of Dresden, Friedrichstrasse, Dresden, Germany
}

Summary. HPV infection is involved in the etiology of a number of nonmalignant, premalignant and malignant cutaneous lesions. One of them is the so-called giant condyloma of Buschke-Löwenstein type (Buschke-Löwenstein tumor, BLT), which sometimes can imitate clinically other tumors or tumor-like conditions. Clinicians face a particular challenge in cases of BLT where, clinically, the lesions demonstrate a permanent brown hyperpigmentation in parallel with the dermatoscopic lack of the characteristic melanocytic network, globules or regression zones. There are uncommon clinical presentations with solitary, sharply demarcated pigmented lesions. In these cases the histopathological verification of the lesion is obligatory and the most efficient treatment method in the early period of the disease is the complete surgical excision. We report a case of a 74-year-old man who was admitted to the University Hospital "Lozenetz" in connection with profuse variceal bleeding of the esophagus associated with liver cirrhosis of unknown etiology. He underwent a consultative examination at the department of dermatology because of suspected advanced stage melanoma of the prepuce. Computed tomographic analysis indicated diffuse bone metastases located in the small pelvis and femur, as well as metastatic disease in the left inguinal lymph nodes. However, the subsequent histopathologic 
examination of the lesion, rather than showing melanoma, confirmed the presence of HPV-associated giant condyloma of Buschke and Löwenstein in initial stage, without histopathological evidence for invasive and destructive tumor growth. After his death, the patient's relatives did not give consent for an autopsy, and therefore the genesis of the metastases, demonstrated by nuclear magnetic resonance imaging (NMR), remained unclear. In some cases, the clinical picture of the malignant and premalignant cutaneous lesions in the genital area could be problematic. The complete surgical excision with a subsequent histopathological verification is the best way to find out the exact diagnosis.

Key words: HPV, papillomatosis, malignant melanoma, differential diagnosis, koilocytosis

\section{INTRODUCTION}

$\mathrm{H}$ uman papilloma virus (HPV) continues to be among the most frequently encountered viruses [1]. The biological cycle of some of these HPV types plays a role in the development of various pre-cancerous and neoplastic processes [2, 3]. In a number of cases, infection with the HPV virus leads to the development of erythroplasia of Queyrat and Bowen's disease and also to the rare manifestation of Buschke-Löwenstein tumor, which affects in most of the cases the penis and surrounding areas and could later progress to invasive squamous cell carcinoma; they can also become sometimes metastatic $[4,5,6]$. Despite the benign histological pattern of giant condyloma acuminatum (GCA) in most cases, transformations into verrucous carcinoma and squamous-cell carcinoma have been described [7].

Giant condyloma acuminatum (Buschke-Lowenstein tumor, BLT) is a human papillomavirus-induced cauliflower-like tumor of the genitoanal region [7]. It is typically characterized by its size, capability of local infiltration and high recurrence rate [7]. Poor hygiene, promiscuity, chronic irritation and immunocompromised states are possible risk factors for its development $[3,7,8]$.

Several studies have tried to elucidate a possible connection between BLT and oncogenic and nononcogenic HPV viruses [1, 2, 3, 9]. We know that it is a rarely seen form which develops as a result of the overgrowth of condyloma acuminatum and is characterized by florid warty growth and aggressive invasion and a risk of malignant transformation $[4,5,6]$.

\section{CASE REPORT}

A 74-year-old patient was transferred to the intensive care ward with a diagnosis of liver cirrhosis of uncertain etiology, anemia, and thrombocytopenia. Upon presentation the patient was in a poor general state, demonstrated by profuse hematemesis, melena, and hemodynamic instability. Though he was communicative at certain moments, at others he was somnolent. In the course of the diagnostic work- 
up, he was found to have complicated liver cirrhosis, esophageal varices, portal hypertension and hepatosplenomegaly. The patient was hospitalized for ten days, after which his status deteriorated further, ending in death as a result of multiple organ failure.

During his stay in the intensive care unit, a dermatology consultation was arranged because of a pigmented lesion in the area of the prepuce, suggestive of penile melanoma, with enlarged left inguinal lymph nodes. Examination revealed a swollen, reddish brown, non-ulcerated plaque on the prepuce measuring $3 \times 1.5$ cm (Fig. 1).

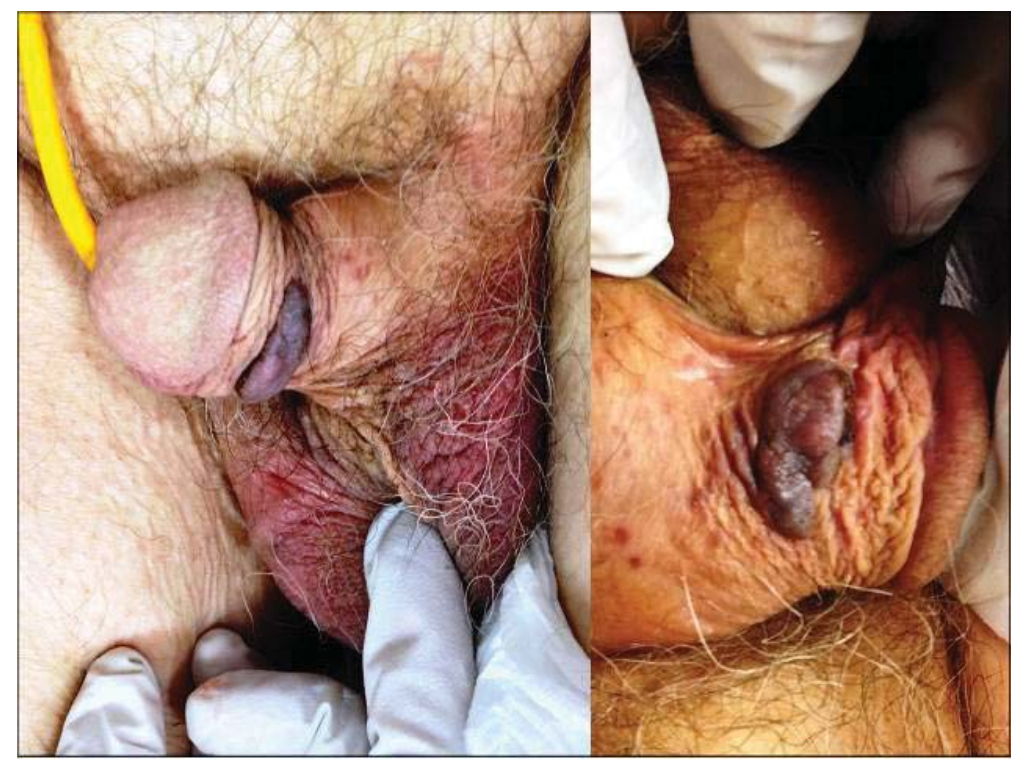

Fig. 1. a-b. HPV-associated giant condyloma of Buschke and Löwenstein mimicking clinically malignant melanoma

\section{DERMATOSCOPICAL EXAMINATION OF THE LESION}

We couldn't observe a peripheral pigment network and oval-shaped globules, which are characteristic for melanocytic lesions. The permanent dark brown color, without the presence of regressive zones (as in our case), was difficult to interpret and demands a skin biopsy or histopathological investigation after surgical excision. This permanent brown color by itself could be observed both in benign and malignant non-melanocytic lesions.

The histopathological findings were consistent with an HPV-associated BLT (Figs. 2a-2d). 


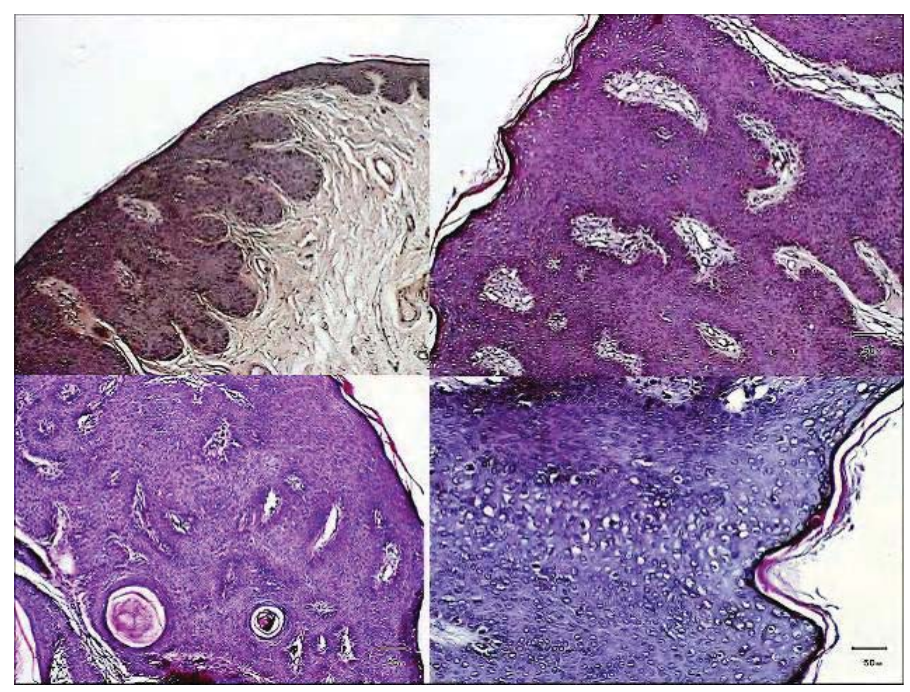

Fig. 2. a-2d. Histopathology of the penile lesion. The epidermis showed parakeratosis, hyperkeratosis, acanthosis resembling pseudoepitheliomatous hyperplasia, papillomatosis, and focal horizontal koilocytic hyperplasia. Within the papillary and reticular dermis there were linear, low grade collagenization and multiple keratin-filled inclusion cysts. Singly distributed melanocytes were found along the junctional zone of the proliferative epidermis. The histopathological findings were consistent with an HPV-associated giant condyloma of Buschke and Löwenstein

\section{IMAGING}

Nuclear magnetic resonance imaging (NMR) revealed a number of enlarged inguinal, pelvic, and para-aortic lymph nodes. Multiple zones of secondary pathologic bone restructuring were found, in addition to hepatosplenomegaly and focal lesions in the spleen suspected of being tumor metastases from an unknown primary site. Endoscopic and x-ray analyses were also performed to evaluate other major disorders and lung pathology. The patient was treated for his serious concomitant diseases (liver cirrhosis, esophageal varices, severe anemia, pyelonephritis) and their complications according to set therapeutic plans.

After his death, the patient's relatives did not consent to an autopsy, and therefore the genesis of his metastases, demonstrated by NMR, remained unclear.

\section{DISCUSSION}

In 1925 Abraham Buschke and Ludwig Loewenstein described a neoplasm of the penis, which, to them, bore resemblances to common condyloma acuminatum and squamous cell carcinoma, but it had histopathological and clinical characteristics that differed from these two proliferations [9]. One of the characteristics of BLT is a benign histopathological pattern (as in our case) that may progress later to extensive papilloma proliferation that deeply penetrates underlying tissues if not 
adequately treated $[4,5,6,9]$. Malignant transformation of BLT has been reported in 30 to $56 \%$ of all cases and the risk of relapses after surgical excision varies between $60-66 \%$ in up to 10 months after the treatment $[7,10]$. In our case the basal layer of the epidermis was intact, so that we excluded a relationship between the inguinal and the intraabdominal metastasis and BLT.

The difference between condyloma acuminata and Buschke-Löwenstein is that BLT has a large and infiltrative base, the possibility of development of a destructive behavior, local relapses and a risk of malignant transformation [4-6, 9]. An important part of the diagnostic work-up are the methods for determining of cell changes specific for HPV infection [1].

Stringent criteria for histological diagnosis of concominant HPV infection are not available [1]. Most condylomas are diagnosed clinically (without a biopsy) or histopathologically (if biopsied) without any ancillary testing. In some cases, additional confirmation of productive infection by human papillomavirus (HPV) or typing of HPV is desired [1].

HPV infection causes koilocytosis [11, 12, 13, 14, 15]. In lesions with histopathologically proven cell atypia additional diagnostic criteria could be also applied: 1) specific autoantiboides against oncogenic and non-oncogenic HPV strains, 2) molecular detection of HPV by DNA-polymerase chain reaction (PCR) or 3) Southern blot hybridization as gold standard and others [1, 15, 16].

We observed koilocytosis in the biopsy as first evidence for the current HPV infection [15]. Koilocytes are squamous epithelial cells that contain an acentric, hyperchromatic nucleus that is displaced by a large perinuclear vacuole. However, the genesis of the cytoplasmic vacuole has remained unclear, particularly because both HPV DNA replication and virion assembly occur exclusively in the nucleus [17]. Additionally, immunohistochemical methods could also be applied, if a possible association with HPV needed further confirmation $[1,16,18]$. For example Anti-HPV antibody was found to be immunoreactive with paraffin sections of formalin-fixed HPV-infected tissues [18]. Occasionally, the cytoplasm of koilocytotic cells was observed to be immunoreactive [18].

And at last, one of the the most authoritative methods for detection of HPV infection is PCR in lesional tissue and the in situ hybridisation $[1,18]$.

Skelton et al. described the case of a 70-year-old patient with a similar keratotic lesion in the area of the buttocks, morphologically identified as endophytic syringocystadenoma papilliferum [19]. Of particular interest is the association made between this lesion and the perilesional epidermis that featured acanthosis, papillomatosis and acantholysis as well as foci containing koilocytes [19]. HPV types 6 and 11 were subsequently found by molecular methods in the nuclei of the altered cells.

Sanclemente et al. described also similar lesions in a patient with black epidermodysplasia verruciformis but with somewhat differing macroscopic characteristics [20]. When evaluating the features of this lesion on the scrotum, the team found alterations with the histopathologic characteristic of an HPV-induced lesion [20]. 
The surgical excision, as in our case, has the advantage of a lower risk of recurrence, it allows a complete histologic examination, the healing process is rapid, the improvement of quality of patient's life is significant $[7,8]$.

Unfortunately, due to the rapid development of multiple organ failure, followed by exitus letalis, the scheduled histopathologic verification of the regional lymph node metastasis became impossible.

\section{REFERENCES}

1. Tchernev, G. Sexually transmitted papillomavirus infections: epidemiology, pathogenesis, clinic, morphology, important differential diagnostic aspects, current diagnostic and treatment options. An. Bras. Dermatol., 84, 2009, № 4, 377-389.

2. Korita, P. V. et al. [Molecular basis of oncogenesis induced by human papilloma viruses]. - Vopr. Onkol., 58, 2012, № 5, 598-605.

3. Henquet, C. J. Anogenital malignancies and pre-malignancies. - J. Eur. Acad. Dermatol. Venereol., 25, 2011, № 8, 885-895.

4. M a n y a m, B. V., M. Feldman et H. Wood. Invasive Penile Buschke-Lowenstein Tumor. - J. Urol., 2013; doi:pii: S0022-5347(13)04865-9. 10.1016/j.juro.2013.07.009.

5. Santos-Rancaño, R. et al. Squamous cell carcinoma arising in a giant condyloma acuminatum - Buschke-Löwenstein cambiar tumor por tumour. - Cir. Esp., 2013, [Epub ahead of print].

6. R a d o va n o vi c, Z. et al. Highly aggressive Buschke-Löwenstein tumor of the perineal region with fatal outcome. - Indian J. Dermatol. Venereol. Leprol., 78, 2012, № 5, 648-650.

7. G h o l a m, P., A. Enk et W. Hartschuh. Successful surgical management of giant condyloma acuminatum (Buschke-Löwenstein tumor) in the genitoanal region: a case report and evaluation of current therapies. - Dermatol., 218, 2009, № 1, 56-59.

8. Tripoli, M. et al. Giant condylomata (Buschke-Löwenstein tumours): our case load in surgical treatment and review of the current therapies. - Eur. Rev. Med. Pharmacol. Sci., 16, 2012, № 6, 747-751.

9. B r a g a , J. C. et al. Loewenstein tumor: identification of HPV type 6 and 11. - An. Bras. Dermatol., 87, 2012, № 1, 131-134.

10. A ye r, J. et al. Successful treatment of Buschke-Lowenstein tumour of the penis with carbon dioxide laser vaporization. - Acta Derm. Venereol., 92, 2012, № 6, 656-657.

11. Gupta, N. et al. Outcome of SurePath ${ }^{\mathrm{TM}}$ cervical samples reported as borderline nuclear change by cytological subtype and high-risk HPV status. - Cytopathology, 23, 2012, № 6, 402-407.

12. Ro eter s, A. M. et al. Papanicolaou's nebular cells are related to HPV infection and HSIL. - Diagn. Cytopathol., 40, 2012, № 3, 197-200.

13. Méndez Toro, L. et al. [HPV infection associated with nonclassic cytological signs in patients from Mérida, Venezuela]. - Invest. Clin., 52, 2011, № 2, 162-169.

14. Alves de Sousa, N. L. et al. Cytopathic effects of human papillomavirus infection and the severity of cervical intraepithelial neoplasia : A frequency study. - Diagn. Cytopathol., 40, 2012, № 10, 871-875.

15. Yumura, Y. et al. [The relationship between human papillomavirus (HPV) infection and penile cancer]. - Hinyokika Kiyo, 55, 2009, № 11, 671-675.

16. Tchernev, G. et al. HPV-associated Buscke-Löwenstein tumours in a patients with disseminated anogenital form of condylomata accuminata - a dermatosurgical problem. - Acta Medica Bulgarica, 2012, № 2, 37-42. 
17. Krawczyk, E. et al. Koilocytosis: a cooperative interaction between the human papillomavirus E5 and E6 oncoproteins. - Am. J. Pathol., 173, 2008, № 3, 682-688.

18. Wititsuwannakul, J. et al. Detecting HPV in cutaneous lesions using anti-HPV antibody immunohistochemistry. - Am. J. Dermatopathol., 35, 2013, № 3, 327-331.

19. Skelton, H. G. 3rd et al. Condyloma acuminatum associated with syringocystadenoma papilliferum. - Am. J. Dermatopathol., 16, 1994, № 6, 628-630.

20. S a n clemente, G. et al. An unusual presentation of human papillomavirus (HPV) infection in a black epidermodysplasia verruciformis (EV) patient. - Int. J. Dermatol., 46, 2007, № 2, 199-201.

$\equiv$ Corresponding author:

Assoc. Prof. Georgi Tchernev

Polyclinic for Dermatology and Venereology, "St. Kliment Ohridski" University of Sofia Medical Faculty

University Hospital Lozenetz

1 Kozyak St.

$\mathrm{Bg}-1407$ Sofia

- 00359885588424

e-mail: georgi_tchernev@yahoo.de 